\title{
A Regression Model for a Displaced Atom Cascade under Ion Sputtering of Solids
}

\author{
V. A. Volpyas and P. M. Dymashevskiü \\ St. Petersburg State University of Electrical Engineering, St. Petersburg, 197376 Russia \\ e-mail: thinfilm@eltech.ru \\ Received October 18, 2000; in final form, February 6, 2001
}

\begin{abstract}
A regression model of an ion-atom collision cascade (and eventually of a displaced atom cascade) resulting under ion sputtering of amorphous and polycrystalline materials is developed. The model allows the description of the elastic scattering of atomic particles in a solid using various particle interaction potentials. Based on this model, we calculate the sputtering yields for materials with atomic numbers $Z_{\mathrm{a}}=22-79$ and the sputtering rates for several multicomponent targets. The results of statistical simulation within the model developed are compared with experimental data for the sputtering of amorphous and polycrystalline materials. It is shown that our model fits the experimental data up to the statistical error and adequately characterizes the ion sputtering process. (C) 2001 MAIK “Nauka/Interperiodica”.
\end{abstract}

\section{INTRODUCTION}

The sputtering of single-component amorphous and polycrystalline targets by ion bombardment is the moststudied field of the physics of material sputtering [1]. It is assumed that the direct knocking-out of atoms from their equilibrium positions in a solid prevails in this case. The available models for ion sputtering of singlecomponent rely on the Sigmund mechanism of physical sputtering by fast ions or atoms [2]. Within this mechanism, the density of a cascade of moving displaced target atoms is described by the distribution function that meets the linearized Boltzmann equation well known from the particle transfer theory. Given the initial velocities and positions of atoms that are decelerated by means of random collisions in an infinite medium, a solution of this equation makes it possible to calculate the atom flux through an arbitrary plane. By taking the target surface as this plane and introducing the parameter $E_{\mathrm{b}}$, the atom-target binding energy, one can determine the escape probability of the displaced atoms, as well as their energy and angular distributions. An elementary event here is a cascade of ion-atom collisions, which causes a displaced atom cascade and makes surface atoms leave the target under certain conditions. Such a sputtering mechanism is considered to be the most probable at ion energies to $1-3 \mathrm{eV}$ and applies to the ion-plasma sputtering of single-components materials.

Today, due to the progress in microelectronics technology, investigations into ion and ion-plasma sputtering of multicomponent materials are becoming more and more urgent. To these materials, sputtering yield as a parameter characterizing the process cannot be applied because of a depleted layer formed on the surface. In this case, the efficiency of sputtering should be described in terms of the target sputtering rate along the depth or a change in the target weight.

The statistical simulation of particle scattering upon ion-atom collisions [3-8] allows researchers to quantitatively estimate the sputtering rate of multicomponent targets. However, the numerical simulation of an ionatom collision cascade and the entire cascade of displaced atoms requires the computer RAM to have an extremely large capacity so as to store huge data arrays of time-varying parameters in different phase spaces (coordinates of cascade particles, their momenta, free path lengths for cascade particles of each sort, etc.). Such a calculation is impossible even with modern computers.

As a reasonable trade-off in this situation, we suggested to mathematically simulate a cascade of ionatom pair collisions with the subsequent description of the bombarding ion trajectory and the energy imparted to target atoms. In essence, this simulation studies individual cascade chains and extends the results to the entire atom-atom collision cascade. Such an approach combines the easy computing procedure (the entire cascade of pair ion-atom collisions and its individual chains are simulated by the Monte Carlo method) and the correct generalization of the simulation results for the displaced atom cascade by regression analysis. This allowed us to develop a regression model for an ionatom collision cascade taking place upon ion sputtering of amorphous and polycrystalline single- and multicomponent materials.

\section{REGRESSION MODEL OF ION-ATOM COLLISION CASCADE}

Consider the form and the application of our model in detail. The trajectory of a bombarding ion in the tar- 
get and momenta gained by target atoms as a result of pair ion-atom collisions are calculated by the Monte Carlo statistical simulation method adapted to particle scattering during ion-atom collisions [9]. When describing the elastic interaction of atomic particles in solids, one must bear in mind the empirical rule according to which collisions causing an appreciable scattering occur at distances on the order of half the equilibrium spacing between adjacent atoms. With so small distances, long-range attracting forces, responsible for binding forces in solids, can be neglected. By order of magnitude, these distances equal the sizes of colliding atomic particles. Therefore, pair ion-atom collisions in solids are usually simulated within the model of quasirigid balls [10], in which the elastic parameters involved in atomic particle scattering are calculated with real potentials of interatomic interaction. Note that this model typically provides relatively high rates of statistical simulation. For a potential of interatomic interaction, we chose the Born-Mayer potential modified [3, 4] for a wide range of colliding partners $\left(Z_{\mathrm{a}}=2-80\right)$.

In a similar way, the trajectories of target atoms of various sort displaced by a bombarding ion (primarily displaced atoms) are simulated and their energy losses in each event of pair atom-atom collision with secondary displaced target atoms are calculated. The trajectories of various branches of the cascade of the primarily displaced atoms are simulated until their energy becomes smaller (because of atom-atom collisions) than the binding energy between atoms of certain sort or until the trajectories cross the target surface. The ratio of the number of displaced atoms in a cascade branch simulated that cross the surface to the number of bombarding ions defines the probability of these atoms escaping the surface, $P_{\mathrm{mc}}$. Its value depends on the atomic weight, atomic number, and initial energy of a bombarding ion, as well as on the atomic weight,

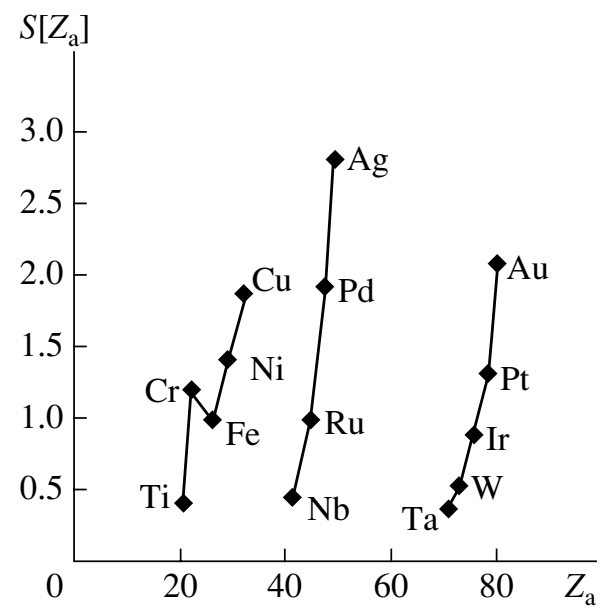

Fig. 1. Sputtering yield in view of the occupancy of the $d$ shell vs. target atomic number $Z_{\mathrm{a}}$ at $\mathrm{Ar}^{+}$bombardment $(E=0.5 \mathrm{keV})[1]$. atomic number, binding energy, and concentration of the target components.

As was noted, the simulation of subsequent branches of the displaced atom cascade is difficult because of the limited capabilities of modern computers. However, regularities obtained in simulating ionatom cascade branches can be extended to all subsequent branches of the displaced atom cascade by using regression analysis. Clearly, the branches of the primarily displaced (by a bombarding ion) atoms involve a greater number of collisions and a higher initial energy than the subsequent atom-atom branches. Therefore, the escape probability for atoms crossing the surface in each of the subsequent atom-atom branches is lower than the simulated value $P_{\mathrm{mc}}$ for the primarily displaced atoms. The smaller the initial energy and the number of atom-atom collisions in any of the secondary branches, the smaller its contribution to the escape probability.

Quantitatively, the ion-induced escape probability $P_{\text {is }}$ (for single-component targets, $P_{\text {is }}$ coincides with the ion sputtering yield $S$; for multicomponent targets, it characterizes the rate of ion sputtering $V_{\text {is }}$ ) can be calculated from the formula

$$
P_{\text {is }}=C_{n}\left(Z_{\mathrm{o}}\right) P_{\mathrm{mc}} W_{\text {ia }} W_{\text {aa }},
$$

where $C_{n}\left(Z_{a}\right)$ is the factor characterizing the shell structure of target atoms, $P_{\mathrm{mc}}$ is the simulated escape probability for a primarily displaced atom (it is calculated by the Monte Carlo method), $W_{\mathrm{ia}}$ is the factor characterizing the degree of regression of the ion-atom collision cascade, and $W_{\text {aa }}$ is the factor that characterizes the degree of regression of the atom-atom collision cascade.

From experimental studies [1], it follows that single-component targets made of Group I materials $(\mathrm{Cu}$, $\mathrm{Ag}$, and $\mathrm{Au}$ ) feature the highest sputtering yield (Fig. 1). These atoms have the occupied $d$ shell $\left(Z_{\mathrm{d}}=\right.$ 18 ), which is assumed to define the binding energy of a target atom. To take into account the shell structure of the atoms in calculating the ion-induced escape probability $P_{\text {is }}$, we approximated the factor $C_{n}\left(Z_{a}\right)$ normalized to $\mathrm{Cu}$ atoms $\left(Z_{\mathrm{a}}=29\right)$ in (1):

$$
C_{n}\left(Z_{\mathrm{a}}\right)=\mid \begin{aligned}
& 0.11 Z_{\mathrm{a}}-2.19 \text { for } Z_{\mathrm{a}}=22-29, \\
& -0.02 Z_{\mathrm{a}}+1.12 \text { for } Z_{\mathrm{a}}=30-41, \\
& 0.216 Z_{\mathrm{a}}-8.56 \text { for } Z_{\mathrm{a}}=41-47 \\
& -0.01 Z_{\mathrm{a}}+1 \text { for } Z_{\mathrm{a}}=48-73, \\
& 0.148 Z_{\mathrm{a}}-10.49 \text { for } Z_{\mathrm{a}}=73-79 .
\end{aligned}
$$

The factors $W_{\mathrm{ia}}$ and $W_{\mathrm{aa}}$ characterizing the degrees of regression of the ion-atom and atom-atom collision cascades were determined with different regression models and checked against experimental data on sputtering yields for a variety of single-component targets and on rates of sputtering multicomponent targets by noble gas ions with various energies. Eventually, we 
obtained

$$
W_{\text {ia }}=\sum_{j=1}^{n_{i}}\left(\frac{j}{n_{i}}\right)^{\left(K_{\mathrm{ri}}-0.5 j / n_{i}\right)},
$$

where $n_{i}$ is the maximal number of collisions of a bombarding ion in the ion-atom collision cascade, $j$ is the serial number of ion-atom collisions $\left(1 \leq j \leq n_{i}\right)$, and $K_{\mathrm{ri}}$ is the regression coefficient for a branch of the ionatom collision cascade, and

$$
W_{\text {aa }}=\sum_{j=1}^{n_{\mathrm{a}}}\left(\frac{j}{n_{\mathrm{a}}}\right)^{\left(K_{\mathrm{ra}}-0.5 j / n_{\mathrm{a}}\right)},
$$

where $n_{a}$ is the maximal number of collisions of a displaced atom in the atom-atom collision cascade, $j$ is the serial number of atom-atom collisions $\left(1 \leq j \leq n_{\mathrm{a}}\right)$, and $K_{\mathrm{ra}}$ is the regression coefficient for a branch of the atom-atom collision cascade.

The regression coefficients $K_{\mathrm{ri}}$ and $K_{\text {ra }}$ were determined by comparing the energy dependence of the sputtering yield $P_{\mathrm{mc}}\left(E_{i}\right)$ obtained from the statistical simulation of the trajectories of the ions and primarily displaced atoms with experimentally found energy dependences of the sputtering yield for $\mathrm{Ti}, \mathrm{Cu}, \mathrm{Nb}, \mathrm{Ag}$, $\mathrm{Ta}$, and Au targets subjected to noble gas ion bombardment [1]. Representing $K_{\text {ri }}$ as a function of the atomic number $Z_{i}$ of a bombarding ion and its energy $E_{i}$, and $K_{\text {ra }}$ as a function of the atomic number of the target $Z_{\mathrm{a}}$ and the energy $E_{i}$ of the bombarding ion in the form

$$
\begin{aligned}
K_{\mathrm{ra}}\left(Z_{\mathrm{a}}, E_{i}\right) & =A\left(Z_{\mathrm{a}}\right) E_{i}+B\left(Z_{\mathrm{a}}\right), \\
K_{\mathrm{ri}}\left(Z_{i}, E_{i}\right) & =C\left(Z_{i}\right) E_{i}+D\left(Z_{i}\right),
\end{aligned}
$$

one can generalize the results and determine the numerical values of the expansion coefficients $A\left(Z_{\mathrm{a}}\right), B\left(Z_{\mathrm{a}}\right)$, $C\left(Z_{i}\right)$, and $D\left(Z_{i}\right)$. The dependences $A\left(Z_{\mathrm{a}}\right)$ and $B\left(Z_{\mathrm{a}}\right)$ for atoms with $Z_{\mathrm{a}}=22-79$ and $\mathrm{Ar}^{+}$ions with energies $E_{i}=$ 250-1000 eV are depicted in Fig. 2.

With such a representation, for the sputtering of targets with $Z_{\mathrm{a}}<80$ by $\mathrm{Ar}^{+}$ions with energies $E_{i}=100$ $1000 \mathrm{eV}, K_{\mathrm{ra}}$ and $K_{\mathrm{ri}}$ are given by

$$
\begin{aligned}
K_{\mathrm{ra}}\left(Z_{\mathrm{a}}, E_{i}\right)= & \left(-2.37 \times 10^{-5} Z_{\mathrm{a}}-0.0016\right) E_{i} \\
+ & \left(0.0705 Z_{\mathrm{a}}+0.3582\right), \\
& K_{\mathrm{ri}}\left(Z_{i}, E_{i}\right)=2.0 .
\end{aligned}
$$

Thus, by calculating the regression coefficients for the cascade branches $K_{\mathrm{ri}}$ and $K_{\mathrm{ra}}$ as functions of given $Z_{i}$ and $E_{i}$ and $Z_{\mathrm{a}}$ and $E_{i}$, respectively, the factors $W_{\mathrm{ia}}$ and $W_{\text {aa }}$, which characterize the degrees of regression for the ion-atom and atom-atom cascades, can be found from (3) and (4). Then, having calculated the escape probability $P_{\mathrm{mc}}$ (the probability of the primarily displaced atom leaving the surface) by the Monte Carlo method and having taken into account the coefficient
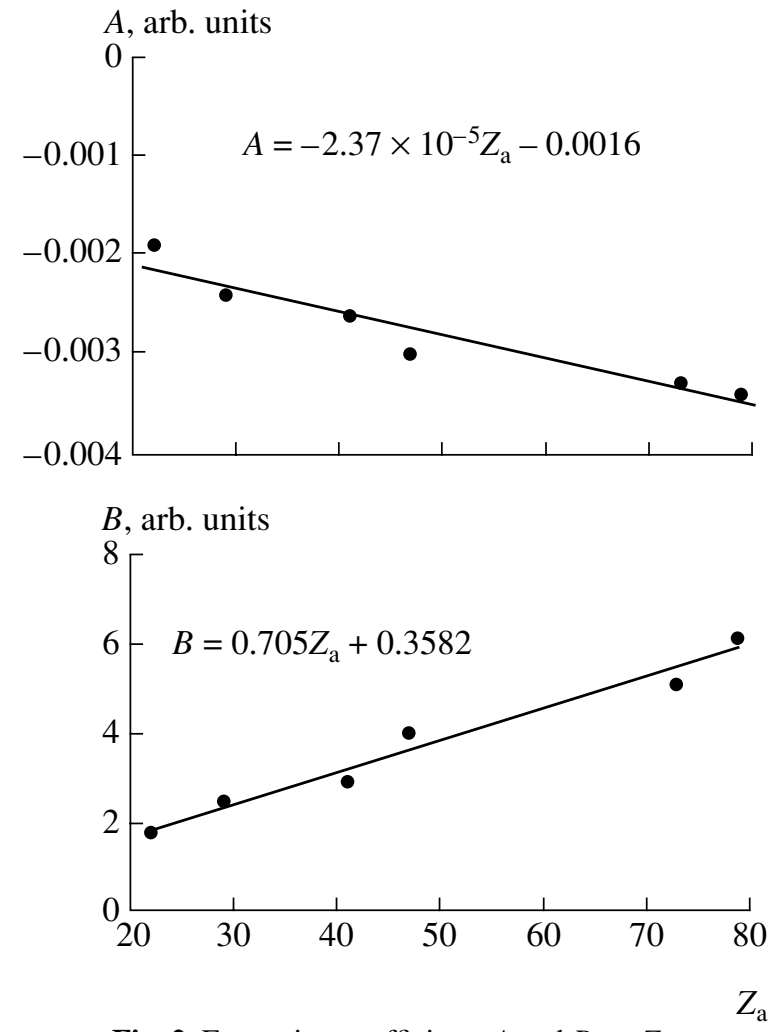

Fig. 2. Expansion coefficients $A$ and $B$ vs. $Z_{\mathrm{a}}$.

$C_{n}\left(Z_{\mathrm{a}}\right)$ [see (2)], characterizing the shell structure of target atoms, one can evaluate the escape probability $P_{\text {is }}$ (the probability of target atoms leaving the surface by the action of a bombarding ion) given by (1). For single-component targets, $P_{\text {is }}$ coincides with the ion sputtering yield $S$; and for multicomponent targets, with the rate of ion sputtering $V_{\text {is }}$. Note that in the latter case, $V_{\text {is }}$ is governed by the sputtering rate of the component having the lowest $P_{\text {is }}$.

\section{DISCUSSION}

Our regression model of ion-atom and atom-atom collision cascades taking place during ion sputtering allows the calculation of the sputtering yields and rates for a wide variety of targets and bombarding ions. Theoretically, the rate of sputtering single-component targets can be estimated from the sputtering yield $S$, which is found within the Sigmund model or with the empirically refined Matsunami formula [11]. However, as follows from the comparison with experimental data [1], the Sigmund model applies at relatively high energies of bombarding ions $\left(E_{i} \geq 1 \mathrm{keV}\right)$, which are of minor importance in ion-plasma sputtering problems. Comparing the results of the statistical simulation in terms of our model with the experimental data for sputtering amorphous and polycrystalline single-component materials shows that our model fairly accurately fits the experimental data (within a statistical experimental 


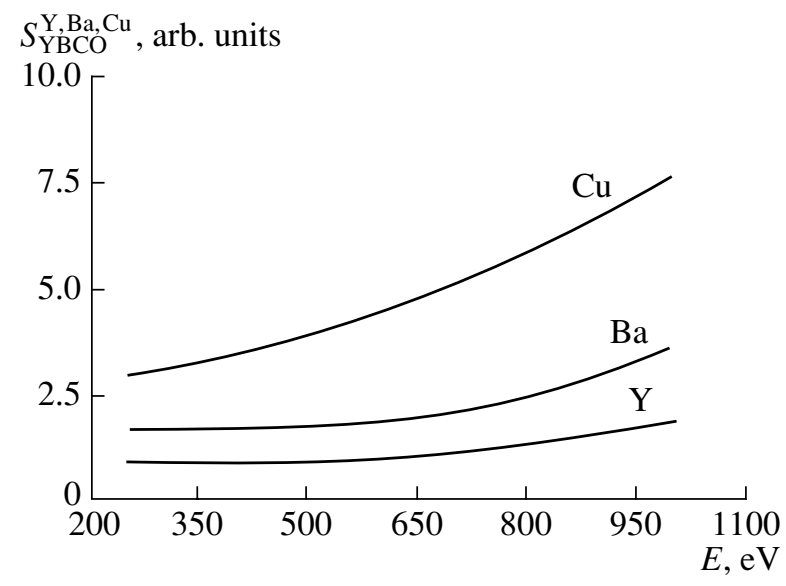

Fig. 3. Sputtering yield for the components of the YBCO ceramic vs. $E$ within the regression model.

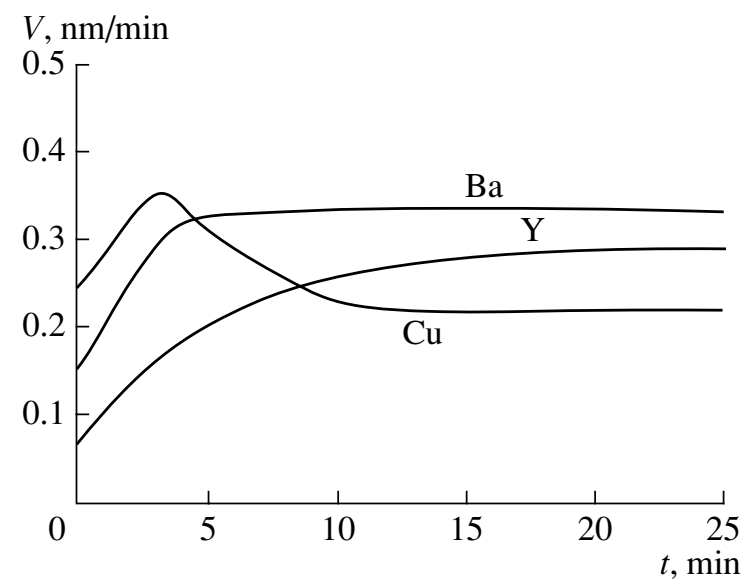

Fig. 4. Sputtering rate $V$ of the YBCO ceramic vs. presputtering time $t$. Sputtering by $\mathrm{Ar}^{+}$ions. The discharge voltage and current density are $160 \mathrm{~V}$ and $4 \mathrm{~mA} / \mathrm{cm}^{2}$, respectively.

error of $10 \%$ [1]) for the sputtering yields and rates in a wide range of bombarding ion energies.

Sputtering of multicomponent targets, specifically, complex oxides of high-temperature superconductors, is a more complicated process than sputtering of singlecomponent targets. Experiments on the sputtering of multicomponent targets [12] show that at the early stage of the process, the composition of the sputtered atomic flux differs from the stoichiometric composition of the target and varies with time. At this stage, the composition of the target surface is other than the stoichiometric composition of the target volume. Due to diffusion processes, the thickness of the nonstoichiometric layer may be about $10 \mathrm{~nm}$. During the process, the composition of the sputtered atom flux becomes constant and coincident with the stoichiometric composition of the target; that is, the sputtering process becomes steady-state. It is known that the greater the bombarding ion flux, i.e., the higher the target sputtering rate, the faster the steady-state regime is set. However, in a number of processes of thin-film evaporation, the sputtering rate is limited by the growth dynamics of a film on a substrate. Therefore, if the evaporation rate is low, the instant the steady-state conditions are established must be detected with a high accuracy.

By way of example, let us consider the sputtering of the high-temperature superconducting ceramic $\mathrm{YBa}_{2} \mathrm{Cu}_{3} \mathrm{O}_{7}$ by $\mathrm{Ar}^{+}$ions and compare our calculations of the sputtering rate with experiments [12]. The calculations show (Fig. 3) that the Y atoms in the YBCO ceramic are the most difficult to sputter: under the conditions of target presputtering, the selective sputtering yield of $\mathrm{Y}$ in the $\mathrm{YBa}_{2} \mathrm{Cu}_{3} \mathrm{O}_{7}$ system $S_{\mathrm{YBCO}}^{\mathrm{Y}}=P_{\text {is }}$ [see (1)] is roughly half as large as those of $\mathrm{Ba}$ and $\mathrm{Cu}$. This is associated largely with the high binding energy of Y, $E_{\mathrm{b}}=20 \mathrm{eV}$ [13]. Thus, the sputtering rate of Y specifies the "sluggishness" of the YBCO target sputtering (Fig. 4), while the $\mathrm{Ba}$ and $\mathrm{Cu}$ atoms have time to adapt to the escape of the $\mathrm{Y}$ atoms from the surface. When ions or atoms bombard targets of complex composition, the kinetic energy of the incident particles is unequally distributed among the components, since atoms with various atomic weights and binding energies participate in the collision cascades. As the energy of incident ions grows, sputtering takes place via collisions between atoms that gained an extra energy from the incident ions rather than directly via ion-atom collisions.

\section{REFERENCES}

1. Sputtering by Particle Bombardment, Ed. by R. Behrisch (Springer-Verlag, New York, 1981, 1983; Mir, Moscow, 1984, 1986), Vols. 1, 2.

2. P. Sigmund, Phys. Rev. 184, 383 (1969).

3. A. A. Abrahamson, Phys. Rev. 178 (3), 76 (1969).

4. V. I. Gaydaenko and V. K. Nikulin, Chem. Phys. Lett. 7, 360 (1970).

5. P. K. Petrov, V. A. Volpyas, E. K. Hollmann, et al., Vacuum 48, 669 (1997).

6. V. A. Volpyas, E. K. Hollmann, D. A. Plotkin, and V. I. Goldrin, Vacuum 51, 227 (1998).

7. V. A. Vol'pyas, E. K. Gol'man, and M. A. Tsukerman, Zh. Tekh. Fiz. 66 (4), 16 (1996) [Tech. Phys. 41, 304 (1996)].

8. V. A. Volpyas and A. B. Kozyrev, Physics of Weakly-Ionized Plasma (Monograph) (Skladen', St. Petersburg, 1997).

9. P. K. Petrov, V. A. Volpyas, and R. A. Chakalov, Vacuum 52, 427 (1999).

10. V. A. Vol'pyas and E. K. Gol'man, Zh. Tekh. Fiz. 70 (3), 13 (2000) [Tech. Phys. 45, 298 (2000)].

11. Y. Yamamura, N. Matsunami, and N. Iton, Radiat. Eff. 71, 65 (1983).

12. V. A. Volpyas, E. K. Hollmann, and V. I. Goldrin, in Abstracts of the 10th International School on Vacuum, Electron and Ion Technologies, Varna, Bulgaria, 1997, p. 18.

13. A. L. Pivovarov, S. P. Chenakin, et al., Poverkhnost 35 (11), 31 (1989).

Translated by V. Isaakyan 